\title{
An Evidence of the Substituent Induced Electronic Interplay. The Effect of the Remote Aromatic Ring Substituent of Phenyl Benzoates on the Sensitivity of the Carbonyl Unit to Electronic Effects of Phenyl or Benzoyl Ring Substituents.
}

\section{Supporting Information}

\author{
Helmi Neuvonen*, Kari Neuvonen and Paavo Pasanen \\ Department of Chemistry, University of Turku, FIN-20014 Turku, Finland
}

\section{List of Contents}

Melting point range and ${ }^{13} \mathrm{C} \mathrm{NMR}$ data $\left(125.78 \mathrm{MHz}, \mathrm{CDCl}_{3}\right)$ for the prepared substituted phenyl benzoates pp. S2 - S4.

$\sigma, \sigma_{\mathrm{F}}$ and $\sigma_{\mathrm{R}}$ values used in the correlations p. S5.

References p. S5. 
Melting point range and ${ }^{13} \mathrm{C}$ NMR data $\left(125.78 \mathrm{MHz}, \mathrm{CDCl}_{3}\right)$ for the prepared substituted phenyl benzoates

4-nitrophenyl 4-nitrobenzoate (1a): $\mathrm{mp} 156.5-157.5^{\circ} \mathrm{C}$ (lit. $\left.{ }^{1,2} 158.5-159.5{ }^{\circ} \mathrm{C}\right),{ }^{13} \mathrm{C}$

NMR $\delta 162.5,155.1,151.2,145.8,133.9,131.5,125.5,123.9,122.5$.

4-cyanophenyl 4-nitrobenzoate (1b): $\mathrm{mp} 190.5-191.5^{\circ} \mathrm{C}$ (lit. ${ }^{2} 189-191{ }^{\circ} \mathrm{C}$ ), ${ }^{13} \mathrm{C} \mathrm{NMR}$ $\delta 162.5,153.6,151.2,134.0,133.9,131.5,123.9,122.7,118.0,110.5$.

4-chlorophenyl 4-nitrobenzoate (1c): $\mathrm{mp} 171.5-172.5^{\circ} \mathrm{C}$ (lit. ${ }^{1} 171-172{ }^{\circ} \mathrm{C}$; lit. ${ }^{2} 171.5-$ $\left.172.5^{\circ} \mathrm{C}\right),{ }^{13} \mathrm{C}$ NMR $\delta 163.1,151.0,148.9,134.6,131.9,131.3,129.8,123.8,122.8$.

4-bromophenyl 4-nitrobenzoate (1d): $\mathrm{mp} 183-184{ }^{\circ} \mathrm{C},{ }^{13} \mathrm{C}$ NMR $\delta$ 163.0, 151.0, 149.5, 134.5, 132.7, 131.3, 123.8, 123.2, 119.6.

phenyl 4-nitrobenzoate (1e): $\mathrm{mp} 127.5-128.0^{\circ} \mathrm{C}$ (lit. $\left.{ }^{1,2} 128-129{ }^{\circ} \mathrm{C}\right),{ }^{13} \mathrm{C}$ NMR $\delta$ $163.3,150.9,150.5,135.0,131.3,129.7,126.4,123.7,121.4$.

4-methylphenyl 4-nitrobenzoate (1f): $\mathrm{mp} 98-99^{\circ} \mathrm{C}$ (lit. $\left.{ }^{1} 98-99{ }^{\circ} \mathrm{C}\right),{ }^{13} \mathrm{C}$ NMR $\delta 163.5$, $150.9,148.3,136.1,135.1,131.3,130.2,123.7,121.06,20.9$.

4-methoxyphenyl 4-nitrobenzoate (1g): $114.0-114.5^{\circ} \mathrm{C}$ (lit. $\left.{ }^{3} 114^{\circ}\right),{ }^{13} \mathrm{C}$ NMR $\delta$ 163.7, 157.7, 150.9, 144.0, 135.1, 131.3, 123.7, 122.2, 114.7, 55.7.

4-nitrophenyl 4-chlorobenzoate (2a): $134.5-135.0{ }^{\circ} \mathrm{C}$ (lit. ${ }^{1} 137-138.5{ }^{\circ} \mathrm{C}$; lit. ${ }^{2} 138-$ $\left.139{ }^{\circ} \mathrm{C}\right),{ }^{13} \mathrm{CNMR} \delta 163.4,155.5,145.5,140.9,131.7,129.2,126.9,125.3,122.6$.

4-cyanophenyl 4-chlorobenzoate (2b): $115-16{ }^{\circ} \mathrm{C},{ }^{13} \mathrm{C}$ NMR $\delta$ 163.5, 154.0, 140.8, $133.8,131.6,129.2,127.1,122.8,118.2,110.0$.

4-chlorophenyl 4-chlorobenzoate (2c): $96.5-96.0{ }^{\circ} \mathrm{C}$ (lit. ${ }^{4} 96{ }^{\circ} \mathrm{C}$, lit. $\left..{ }^{1} 96-96.5^{\circ} \mathrm{C}\right),{ }^{13} \mathrm{C}$ NMR $\delta 164.1,149.2,140.4,131.5,131.6,129.6,129.0,127.6,123.0$.

4-bromophenyl 4-chlorobenzoate (2d): $\mathrm{mp} 102-104{ }^{\circ} \mathrm{C}$ (lit. ${ }^{5} 100-104{ }^{\circ} \mathrm{C}$; lit. $^{6} 105-$ $\left.106{ }^{\circ} \mathrm{C}\right),{ }^{13} \mathrm{C}$ NMR $\delta 164.0,149.8,140.4,132.6,131.6,129.0,127.6,123.4,119.2$. phenyl 4-chlorobenzoate (2e): $\mathrm{mp} 103-104{ }^{\circ} \mathrm{C}$ (lit. ${ }^{1} 103.5-104.5$; lit. $^{5} 100.5-101.5$ $\left.{ }^{\circ} \mathrm{C}\right),{ }^{13} \mathrm{C}$ NMR $\delta 164.3,150.8,140.2,131.5,129.5,129.0,128.0,126.0,121.6$. 4-methylphenyl 4-chlorobenzoate (2f): $\mathrm{mp} 96.0-96.5^{\circ} \mathrm{C}$ (lit. ${ }^{1} 97.5-98.5^{\circ} \mathrm{C}$; lit. ${ }^{4} 98$ $\left.{ }^{\circ} \mathrm{C}\right),{ }^{13} \mathrm{C}$ NMR $\delta 164.5,148.5,140.0,135.7,131.5,130.1,128.9,128.1,121.3,20.9$. 
4-methoxyphenyl 4-chlorobenzoate (2g): $\mathrm{mp} 101-102{ }^{\circ} \mathrm{C},{ }^{13} \mathrm{C} \mathrm{NMR} \delta 164.7,157.4$, $144.2,140.0,131.5,128.9,128.1,122.4,114.6,55.6$.

4-nitrophenyl benzoate (3a): mp $118.5-119^{\circ} \mathrm{C}$ (lit. ${ }^{1,2} 142-143{ }^{\circ} \mathrm{C}$; lit. $\left.{ }^{4} 142{ }^{\circ} \mathrm{C}\right){ }^{13} \mathrm{C}$ NMR $\delta 164.2,155.7,145.4,134.3,130.3,128.8,128.5,125.3,122.6$.

4-cyanophenyl benzoate (3b): mp 91.0-91.5 ${ }^{\circ} \mathrm{C}$ (lit. $\left.{ }^{7} 91-92{ }^{\circ} \mathrm{C}\right),{ }^{13} \mathrm{C} \mathrm{NMR} \delta 164.3$, $154.3,134.2,133.7,130.3,128.8,128.7,122.9,118.3,109.8$.

4-chlorophenyl benzoate (3c): $\mathrm{mp} 88-89{ }^{\circ} \mathrm{C}$ (lit. $.{ }^{1} 88-89{ }^{\circ} \mathrm{C}$; lit. ${ }^{7} 88-90{ }^{\circ} \mathrm{C}$ ), ${ }^{13} \mathrm{C}$ NMR $\delta 164.9,149.4,133.8,131.3,130.2,129.5,129.2,128.6,123.1$.

4-bromophenyl benzoate (3d): mp $92-93{ }^{\circ} \mathrm{C}\left(\right.$ lit. $\left.^{5} 104-106{ }^{\circ} \mathrm{C}\right),{ }^{13} \mathrm{C}$ NMR $\delta 164.9$, $150.0,133.8,132.5,130.2,129.2,128.6,123.5,119.0$.

phenyl benzoate (3e): $\mathrm{mp} 69-70{ }^{\circ} \mathrm{C}$ (lit. $\left.{ }^{7} 69-70{ }^{\circ} \mathrm{C}\right),{ }^{13} \mathrm{C}$ NMR $\delta$ 165.2, 151.0, 133.6, $130.2,129.5,129.6,128.6,125.9,121.7$.

4-methylphenyl benzoate (3f): $\mathrm{mp} 71-72{ }^{\circ} \mathrm{C}$ (lit. ${ }^{1} 70.4-71.2{ }^{\circ} \mathrm{C}$; lit. $\left.{ }^{5} 70-71{ }^{\circ} \mathrm{C}\right),{ }^{13} \mathrm{C}$ NMR $\delta 165.4,148.7,135.5,133.5,130.2,130.0,129.7,128.5,121.4,20.9$.

4-methoxyphenyl benzoate (3g): $\mathrm{mp} 87-88^{\circ} \mathrm{C}\left(\right.$ lit. $\left.^{5} 84-86{ }^{\circ} \mathrm{C}\right),{ }^{13} \mathrm{C}$ NMR $\delta$ 165.6, $157.3,144.4,133.5,130.1,129.7,128.5,122.5,114.5,55.6$.

4-nitrophenyl 4-methylbenzoate (4a): mp $118.5-119.5^{\circ} \mathrm{C}$ (lit. ${ }^{1} 120.3-121.3{ }^{\circ} \mathrm{C}$; lit. $^{2}$ 119.5-120 $\left.{ }^{\circ} \mathrm{C}\right),{ }^{13} \mathrm{C}$ NMR $\delta 164.3,155.9,145.33,145.28,130.4,129.5,125.8,125.2$, $122.7,21.8$.

4-cyanophenyl 4-methylbenzoate (4b): $\mathrm{mp} 142-143{ }^{\circ} \mathrm{C},{ }^{13} \mathrm{C}$ NMR $\delta 164.4,154.4$, $145.2,133.7,130.3,129.5,125.9,123.0,118.3,109.7,21.8$.

4-chlorophenyl 4-methylbenzoate (4c): mp 89-90 ${ }^{\circ} \mathrm{C}$ (lit. ${ }^{1} 89.2-90.7{ }^{\circ} \mathrm{C}$; lit. $^{4} 91{ }^{\circ} \mathrm{C}$ ), ${ }^{13}$ C NMR $\delta 165.0,149.5,144.7,131.2,130.2,129.5,129.4,126.4,123.2,21.8$. 4-bromophenyl 4-methylbenzoate (4d): mp 103-104 ${ }^{\circ} \mathrm{C}\left(\right.$ lit. $\left.{ }^{5} 94-98{ }^{\circ} \mathrm{C}\right),{ }^{13} \mathrm{C}$ NMR $\delta$ $164.9,150.0,144.7,132.5,130.2,129.4,126.4,123.6,118.9,21.8$.

phenyl 4-methylbenzoate (4e): mp 72.5-73.0 ${ }^{\circ} \mathrm{C}$ (lit. $.^{1} 75-76.5^{\circ} \mathrm{C}$; lit. ${ }^{5} 70.5-72.5^{\circ} \mathrm{C}$ ), ${ }^{13} \mathrm{C}$ NMR $\delta 165.3,151.0,144.4,130.2,129.5,129.3,126.8,125.8,121.8,21.8$. 
4-methylphenyl 4-methylbenzoate (4f): mp 89.0-89.5 ${ }^{\circ} \mathrm{C}$ (lit. $\left.{ }^{1} 89.0-90.5{ }^{\circ} \mathrm{C}\right),{ }^{13} \mathrm{C}$ NMR $\delta 165.4,148.8,144.3,135.4,130.2,130.0,129.2,126.9,121.4,21.7,20.9$.

4-methoxyphenyl 4-methylbenzoate (4g): mp 93-94 ${ }^{\circ} \mathrm{C}\left(\right.$ lit. $\left.{ }^{9} 93-95{ }^{\circ} \mathrm{C}\right),{ }^{13} \mathrm{C}$ NMR $\delta$ $165.6,157.3,144.5,144.3,130.2,129.3,126.9,122.5,114.5,55.6,21.8$.

4-nitrophenyl 4-methoxybenzoate (5a): $\mathrm{mp} 164.5-165.5{ }^{\circ} \mathrm{C}$ (lit. ${ }^{7} 164-165{ }^{\circ} \mathrm{C}$; lit. ${ }^{2} 165$ $\left.166{ }^{\circ} \mathrm{C}\right),{ }^{13} \mathrm{C}$ NMR $\delta 164.4,163.9,155.9,145.3,132.5,125.2,122.7,120.7,114.1,55.6$.

4-cyanophenyl 4-methoxybenzoate (5b): $\mathrm{mp} 109-110{ }^{\circ} \mathrm{C},{ }^{13} \mathrm{C} \mathrm{NMR} \delta 164.3,164.1$, $154.4,133.7,132.5,123.0,120.9,118.4,114.0,109.6,55.6$.

4-chlorophenyl 4-methoxybenzoate (5c): mp $92.5-93.0{ }^{\circ} \mathrm{C}\left(\right.$ lit. $\left.^{2} 91.5-92.5{ }^{\circ} \mathrm{C}\right),{ }^{13} \mathrm{C}$ NMR $\delta 164.7,164.1,149.5,132.4,131.1,129.5,123.2,121.5,113.9,55.5$.

4-bromophenyl 4-methoxybenzoate (5d): mp $104-105^{\circ} \mathrm{C}\left(\right.$ lit. $\left.^{5} 103-104.5^{\circ} \mathrm{C}\right),{ }^{13} \mathrm{C}$ NMR $\delta 164.6,164.1,150.1,132.5,132.3,123.6,121.4,118.8,113.9,55.5$.

phenyl 4-methoxybenzoate(5e): $\mathrm{mp} 72-73{ }^{\circ} \mathrm{C}$ (lit. ${ }^{5} 67.5-69{ }^{\circ} \mathrm{C}$; lit. $\left.{ }^{8} 73.6{ }^{\circ} \mathrm{C}\right),{ }^{13} \mathrm{C} \mathrm{NMR}$ $\delta 164.9,163.9,151.1,132.3,129.4,125.7,121.9,121.8,113.8,55.5$.

4-methylphenyl 4-methoxybenzoate (5f): $\mathrm{mp} 66-67{ }^{\circ} \mathrm{C}\left(\right.$ lit. $\left.{ }^{8} 66.9{ }^{\circ} \mathrm{C}\right),{ }^{13} \mathrm{C} \mathrm{NMR} \delta$ $165.1,163.8,148.8,135.3,132.3,129.9,122.0,121.5,113.8,55.5,20.9$.

4-methoxyphenyl 4-methoxybenzoate (5g): mp $124.0-124.5{ }^{\circ} \mathrm{C},{ }^{13} \mathrm{C}$ NMR $\delta$ 165.3, $163.8,157.2,144.5,132.2,122.5,122.0,114.5,113.8,55.6,55.5$.

4-nitrophenyl 4-dimethylaminobenzoate (6a): mp 195-197 ${ }^{\circ} \mathrm{C}$ (dec.) (lit. ${ }^{1} 195-196.5$

$\left.{ }^{\circ} \mathrm{C}\right),{ }^{13} \mathrm{C}$ NMR $\delta 164.4,156.4,154.1,145.0,132.2,125.1,122.7,114.6,110.8,40.1$.

4-cyanophenyl 4-dimethylaminobenzoate (6b): $\mathrm{mp} 152-153{ }^{\circ} \mathrm{C},{ }^{13} \mathrm{C} \mathrm{NMR} \delta 164.6$, $154.9,154.0,133.6,132.2,123.1,118.6,114.7,110.8,109.1,40.1$.

4-chlorophenyl 4-dimethylaminobenzoate (6c): mp $134-135{ }^{\circ} \mathrm{C},{ }^{13} \mathrm{C}$ NMR $\delta 165.2$, $153.8,149.9,132.0,130.7,129.3,123.4,115.4,110.8,40.0$.

4-bromophenyl 4-dimethylaminobenzoate (6d): $\mathrm{mp} 142.5-143.5{ }^{\circ} \mathrm{C},{ }^{13} \mathrm{C} \mathrm{NMR} \delta$ $165.2,153.8,150.4,132.3,132.0,123.8,118.4,115.4,110.7,40.0$. phenyl 4-dimethylaminobenzoate (6e): mp $179.5-180.5^{\circ} \mathrm{C}$ (lit. $\left.{ }^{1} 180-181^{\circ} \mathrm{C}\right),{ }^{13} \mathrm{C}$ NMR $\delta 165.5,153.7,151.4,132.0,129.3,125.4,122.0,116.0,110.8,40.1$. 
4-methylphenyl 4-dimethylaminobenzoate (6f): mp $160-161{ }^{\circ} \mathrm{C}$ (dec.) (lit. ${ }^{1} 160-161$

$\left.{ }^{\circ} \mathrm{C}\right),{ }^{13} \mathrm{C}$ NMR $\delta 165.7,153.7,149.1,134.9,131.9,129.8,121.6,116.1,110.7,40.1,20.9$. 4-methoxyphenyl 4-dimethylaminobenzoate (6g): $\mathrm{mp} 173.0-173.5{ }^{\circ} \mathrm{C},{ }^{13} \mathrm{C} \mathrm{NMR} \delta$ $165.9,157.0,153.7,144.8,131.9,122.7,116.1,114.4,110.7,55.6,40.1$.

$\sigma, \sigma_{F}$ and $\sigma_{R}$ values used in the correlations

$\begin{array}{llll}\text { Substituent } & \sigma^{a} & \sigma_{\mathrm{F}}{ }^{b} & \sigma_{\mathrm{R}}{ }^{b} \\ \mathrm{NO}_{2} & 0.78 & 0.65 & 0.18 \\ \mathrm{CN} & 0.66 & 0.60 & 0.10 \\ \mathrm{Cl} & 0.23 & 0.45 & -0.17 \\ \mathrm{Br} & 0.23 & 0.45 & -0.16 \\ \mathrm{H} & 0 & 0 & 0 \\ \mathrm{Me} & -0.17 & 0 & -0.08 \\ \mathrm{OMe} & -0.27 & 0.25 & -0.42 \\ \mathrm{NMe}_{2} & -0.83 & 0.10 & -0.64\end{array}$

${ }^{a}$ Ref. 10. ${ }^{b}$ Ref. 11, except for Br ref. 10.

\section{References for the Supporting Information}

(1) Kirsch, J. F.; Clewell, W.; Simon, A. J. Org. Chem. 1968, 33, 127.

(2) Menger, F. M.; Smith, J. H. J. Am. Chem. Soc. 1972, 94, 3824.

(3) Satchell, D. P. N.; Secemski, I. I. J. Chem. Soc. B. 1969, 130.

(4) Shawali, A. S.; Eweiss, N. F. Can J. Chem. 1977, 55, 3967.

(5) Hashimoto, S.; Furukawa, I. Bull. Chem. Soc. Jpn. 1981, 54, 2227.

(6) Cadogan, J. I. G.; Smith, D. M.; Thomson; J. B. J. Chem. Soc., Perkin Trans. 1, 1972, 1296.

(7) Correia, V. R.; Cuccovia, I. M.; Chaimovich, H. J.Phys. Org. Chem. 1991, 4, 13.

(8) Martin, R.; Gros, N.; Böhmer, V.; Kämmerer, H. Monatsh. Für Chemie 1979, $110,1057$. 
(9) Engbersen, J. F. J.; Geurtsen, G.; DeBie D. A.; Van der Plas, H. C. Tetrahedron 1968, 44, 1795.

(10) Hansch, C.; Leo, A.; Taft, R. W. Chem. Rev. 1991, 91, 165.

(11) Taft, R. W.; Topsom, R. D. Prog. Phys. Org. Chem. 1987, 16, 1. 